\title{
April 2015 Imaging Case of the Month
}

\author{
Michael B. Gotway, MD \\ Department of Radiology \\ Mayo Clinic Arizona \\ Scottsdale, AZ
}

Clinical History: A 73-year-old woman with a history of ovarian malignancy in remission for several years and treated with hysterectomy and oopherectomy, hypothyroidism, and hypertension, presented with rather abrupt onset cough, chest pain, dyspnea and low-grade fever $\left(99.6^{\circ} \mathrm{F}\right)$. Her past medical history was otherwise unremarkable. Her medications included thyroid replacement, amlodipine, benazepril, and, recently, calcium and magnesium supplementation. Chest radiography was performed (Figure 1).

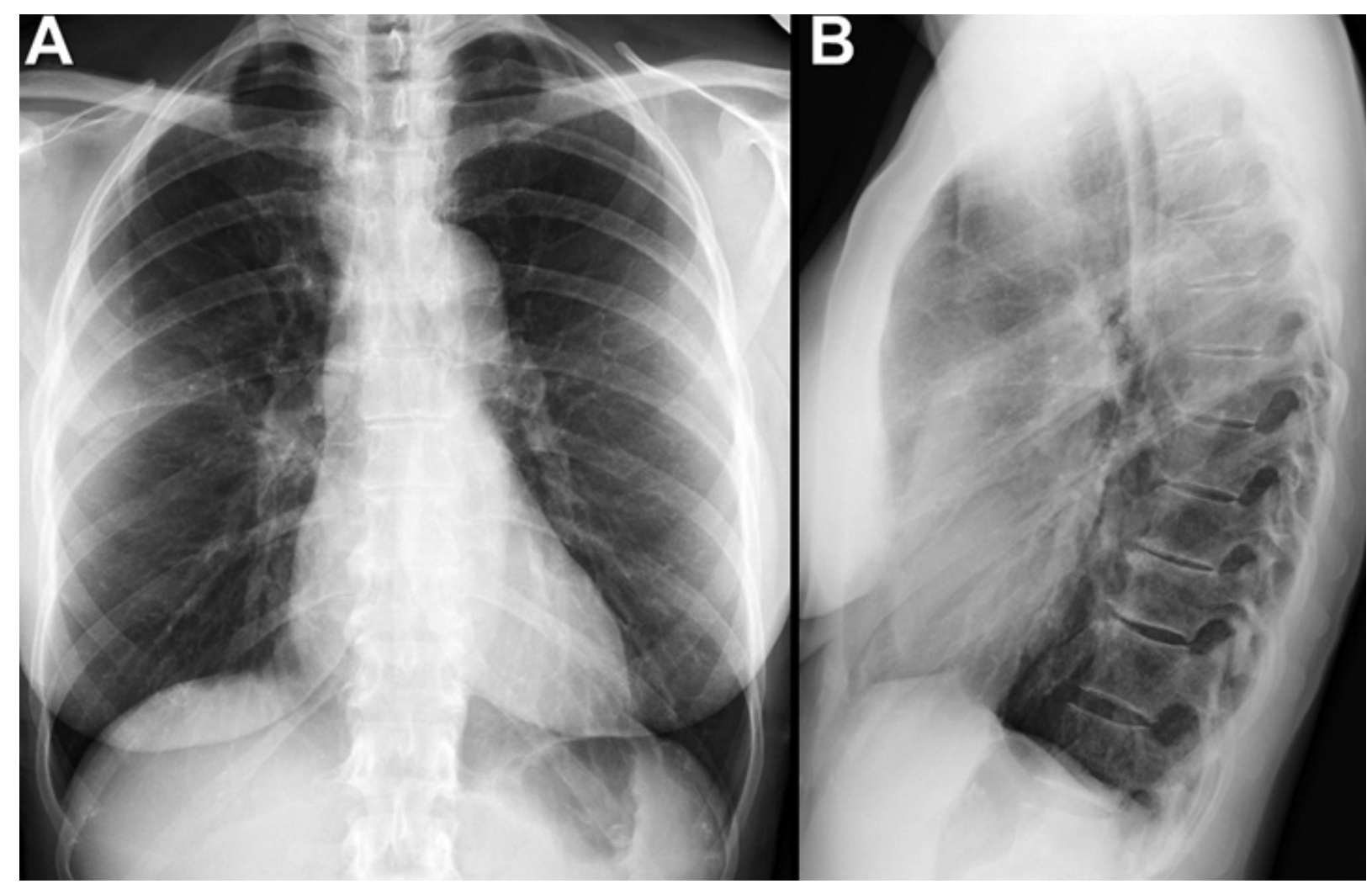

Figure 1. Frontal (panel A) and lateral (panel B) chest radiographs.

Which of the following statements regarding the chest radiograph is most accurate?

1. Chest radiography shows a large right pleural effusion

2. Chest radiography shows focal lung opacity

3. Chest radiography shows mediastinal widening

4. Chest radiography shows pneumomediastinum

5. Chest radiography shows pneumothorax 


\section{Correct!}

\section{Chest radiography shows focal lung opacity}

The chest radiograph shows focal, peripheral right lung hazy opacification. There is no evidence of pneumothorax or pneumomediastinum. The mediastinum shows normal width and contour, with a tortuous aorta. No pleural abnormalities are seen.

Which of the following represents the most likely diagnosis for this patient?

1. Metastatic ovarian malignancy

2. Pneumonia

3. Pneumonitis related to ovarian malignancy treatment

4. Pulmonary contusion

5. Pulmonary embolism 


\section{Correct!}

\section{Pneumonia}

In outpatients who present for thoracic imaging with relatively acute onset of respiratory symptoms, pulmonary infection is the most likely cause for a poorly defined focal pulmonary opacity. The ill-defined focal nature of the process at chest radiography would be a highly unusual manifestation of ovarian malignancy. Ovarian malignancy often presents in the thorax with pleural abnormalities, or perhaps lymph node enlargement or lung nodules, typically in the context of subdiaphragmatic disease. Pneumonitis related to radiotherapy, with or without concomitant chemotherapy, may present with focal lung opacity, but the history specifically states that the patient's ovarian malignancy is in remission, so this potential etiology is not a consideration. Pulmonary contusion often presents with peripheral lung opacity, but no history of thoracic trauma is given. Finally, pulmonary abnormalities in the context of thromboembolic disease, such as atelectasis, pulmonary hemorrhage, or pulmonary infarction, may present with peripheral lung opacities, but such processes are less common than pulmonary infection).

Clinical course: Previous chest radiographs performed approximately 1 year earlier (Figure 2) were obtained for comparison.

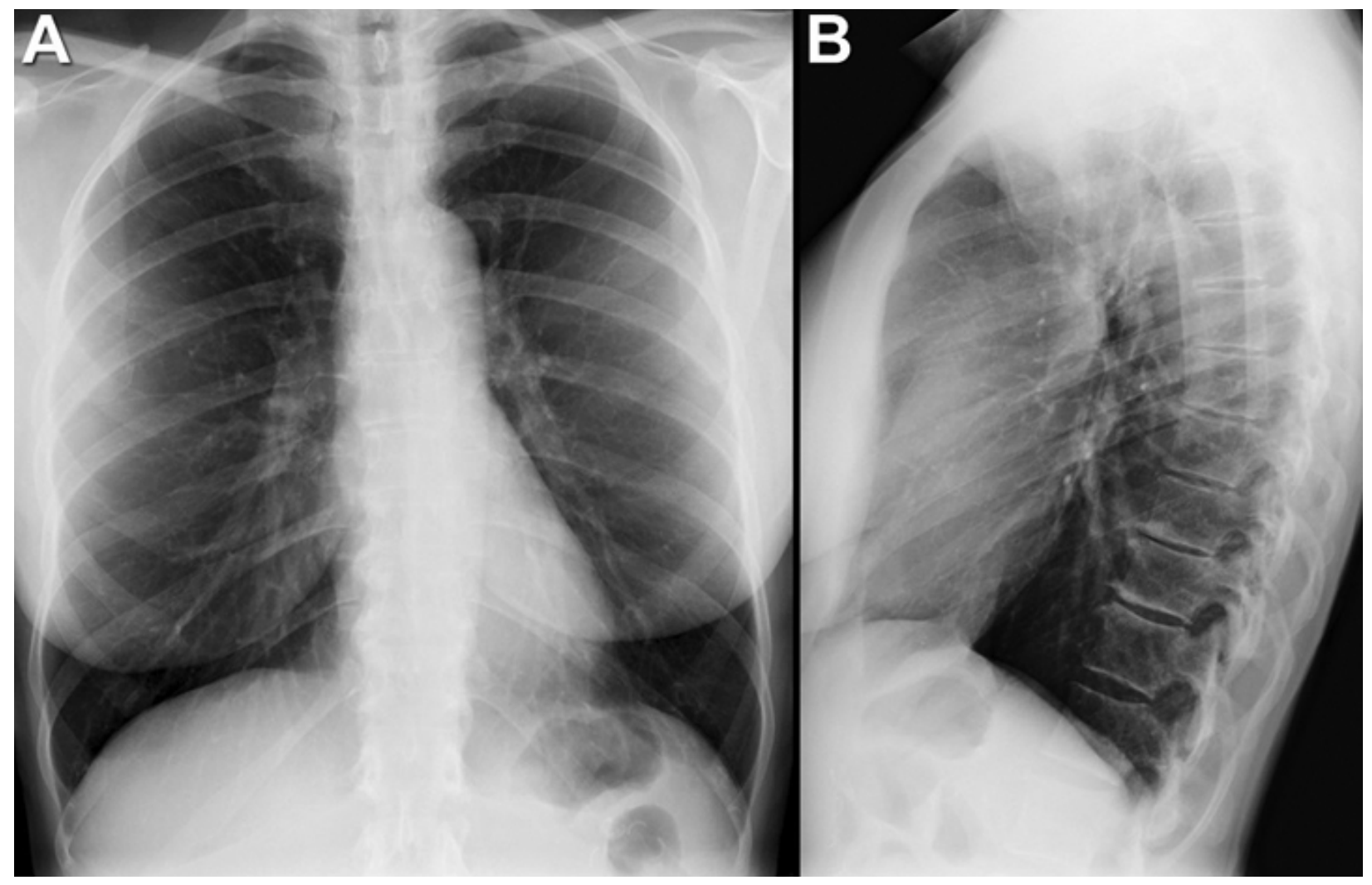

Figure 2. Frontal and lateral chest radiographs performed over 1 year prior to presentation imaging (Figure 1). 
Which of the following statements regarding the chest radiograph is most accurate?

1. Comparison to previous chest radiography shows a nodule on the prior study in the location of the peripheral right lung abnormality currently

2. Comparison to previous chest radiography shows migratory bilateral opacities

3. Comparison to previous chest radiography shows the right lung findings persist unchanged

4. Comparison to previous chest radiography shows the right lung findings are new

5. Comparison to previous chest radiography shows the right lung findings are worsening from previous 


\section{Correct!}

4. Comparison to previous chest radiography shows the right lung findings are new

The previous chest radiograph (Figure 2) shows normal findings. The right lung opacity detected at presentation imaging (Figure 1) is new; no nodule or other abnormality was present in this region previously. The left lung was previously clear and therefore no evidence of migratory opacities is seen).

Which of the following represents the next most appropriate step for the evaluation of this patient?

1. ${ }^{68} \mathrm{Ga}$-citrate scintigraphy

2. ${ }^{99 \mathrm{~m}} \mathrm{Tc}-\mathrm{MAA}$ ventilation-perfusion scintigraphy

3. Decubitus chest radiography

4. Thoracic MRI

5. Treatment for presumed community-acquired pneumonia with imaging follow up to show resolution 


\section{Correct! \\ 5. Treatment for presumed community-acquired pneumonia with imaging follow up to show resolution}

Absent other atypical clinical features or extenuating circumstances, it is appropriate to treat a presumed community-acquired pneumonia and re-evaluate the patient following treatment. The time interval required for imaging follow up to show clearing of a presumed pneumonia detected at chest radiography is variable. In older patients, it is not uncommon for opacities related to pulmonary infection detected at imaging to be visualized for several months, whereas in other patients, sometimes the opacities will resolve quickly. Typically repeat imaging is not required for several weeks at least, unless clinical symptoms worsen or assessment suggests the original diagnosis was incorrect or a complication may be developing. ${ }^{99 m}$ Tc-MAA ventilation-perfusion scintigraphy is useful for the detection of suspected thromboembolic disease and is not a "wrong" answer, but pulmonary embolism is not highly suspected in this patient and therefore ${ }^{99 \mathrm{~m}}$ Tc-MAA ventilation-perfusion scintigraphy is not the most appropriate next step. Thoracic MRI plays essentially no role in the assessment of patients with focal pulmonary opacities- the signal obtained from lung at thoracic MRI is rather poor and when advanced imaging is needed, CT is faster, better tolerated, and generally more rewarding. ${ }^{68} \mathrm{Ga}$-citrate scintigraphy is occasionally used for assessment of diffuse lung opacities, perhaps to detect opportunistic infections in immunocompromised patients, or pneumonitis related to medication-induced pulmonary injury, but neither are appropriate considerations for this patient. Decubitus chest radiography is useful for detection of pleural fluid (on the dependent side) or pneumothorax (on the non-dependent side), but neither complication is suspected in this case).

Further clinical course: Over the next few days that patient complained of worsening cough and blood-streaked sputum.

Which of the following represents the next most appropriate step for the evaluation of this patient?
1. ${ }^{18}$ FDG-PET
2. ${ }^{99 \mathrm{~m}} \mathrm{Tc}-\mathrm{MAA}$ ventilation-perfusion scintigraphy
3. Catheter pulmonary angiography
4. Thoracic CT
5. Thoracic MRI 


\section{Correct! \\ 4. Thoracic CT}

The persistent and worsening clinical symptoms, particularly with the development of blood-streaked sputum, raise the possibility of thromboembolic disease, so ${ }^{99 \mathrm{~m}} \mathrm{Tc}-\mathrm{MAA}$ ventilation-perfusion scintigraphy is not an incorrect step. However, anatomic imaging would probably be more efficacious in this setting as the patient is complaining primarily of worsening cough. Therefore, thoracic CT is the best choice, and could be performed as CT pulmonary angiography if desired to simultaneously exclude thromboembolic disease. Again, thoracic MRI does not play a role for the evaluation of patients with new-onset respiratory symptoms, such as cough, particularly in the acute setting. Thoracic MRI may have some role in the staging of lung malignancies and assessment of mediastinal masses, and perhaps in the pre-operative staging of malignant pleural mesothelioma, but offers little value in the assessment of pulmonary disorders that cannot otherwise be gleaned from chest radiography or CT. ${ }^{18}$ FDG-PET plays an important role in the evaluation of solitary pulmonary nodules and lung cancer staging, but focal opacities suspicious for pneumonia are not readily assessed with this technique because tracer avidity can occur with either inflammatory or neoplastic lesions, whereas the lack of tracer utilization in a focal pulmonary opacity, in a symptomatic patient, provides little management-altering information. Catheter pulmonary angiography has a very limited role in modern medical practice, and is used primarily for treatment of arteriovenous malformations or interventions related to pulmonary embolism, or, even less commonly, pre-operative embolization of congenital I developmental lesions, such as sequestrations, but has no role in the evaluation of this patient).

Further clinical course: The patient underwent unenhanced thoracic CT (Figure 3), with a thoracic CT performed over one year previously (Figure 4) presented for comparison.

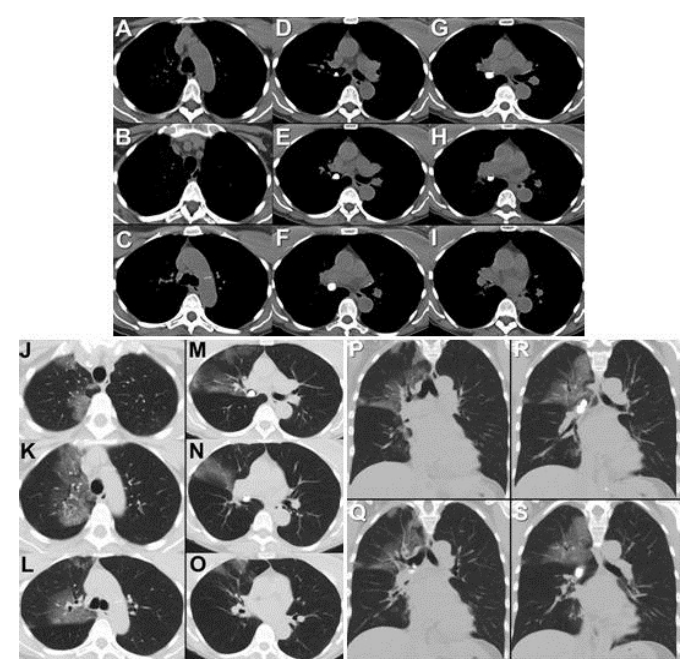

Figure 3. Panels A-I: Axial unenhanced thoracic CT displayed in soft tissue windows. Panels J-O: Axial unenhanced thoracic CT displayed in lung windows. Panels P-S: Coronal unenhanced thoracic CT displayed in a wide window width. 


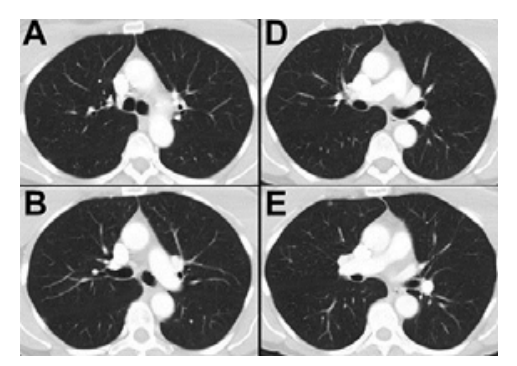

Figure 4. Axial unenhanced thoracic CT displayed in lung windows performed 1 year prior to Figure 3.

Which of the following statements regarding the thoracic CT is most accurate?

1. The thoracic CT shows abnormal findings, but these abnormalities were also present on the comparison CT over one year earlier

2. The thoracic CT shows findings less extensive than those seen at chest radiography, suggesting the process is already resolving

3. The thoracic CT shows findings not readily appreciated at the presentation chest radiograph

4. The thoracic CT shows the same findings seen at chest radiography but discloses no new or management-altering information

5. The unenhanced nature of the CT examination renders it too limited to be of diagnostic value 


\section{Correct! \\ 3. The thoracic CT shows findings not readily appreciated at the presentation chest radiograph}

The thoracic CT shows findings that were seen at presentation chest radiographynamely the right lung opacity- and that the opacity is at least similar in extent to the abnormality identified at chest radiography. However, the thoracic CT shows additional information that is not readily visualized on the initial chest radiograph (Figure 1), and therefore is not only technically adequate but diagnostically useful. The abnormalities seen at the recent CT (Figure 3) are new from the prior CT one year earlier (Figure 4).

Based on the CT findings and persistent, even worsening clinical symptoms, which of the following represents the next most appropriate step for the evaluation of this patient?

1. Bronchial arteriography

2. Bronchoscopy

3. Prone, decubitus, and dynamic expiratory thoracic CT

4. Upper endoscopy

5. Virtual bronchoscopy 


\section{Correct! \\ 2. Bronchoscopy}

Bronchoscopy represents the next most appropriate step for this patient as the thoracic CT clearly shows the lesion(s) to reside within the proximal airways. Upper endoscopy is not appropriate for this patient as the abnormalities seen at thoracic CT are endobronchial, not within the esophagus or periesophageal mediastinum. Bronchial arteriography is primarily used as part of an embolization procedure for patients with hemoptysis, often in the setting of chronic inflammatory pulmonary diseases. While this patient does have blood-streaked sputum, the nature this complaint is insufficient to prompt consideration for bronchial arteriography. Prone CT is often used in patients with suspected interstitial lung disease to determine if basal lung opacities seen at supine thoracic CT persist, which would suggest fibrotic lung disease or alveolitis over dependent atelectasis, but that consideration is not relevant to this patient. Decubitus and dynamic expiratory CT are both techniques that can be used to assess for air trapping, typically for the detection of small airway obstruction. Given the endobronchial right mainstem bronchial and bronchus intermedius location of the hyperattenuating opacities, air trapping may occur in this patient if decubitus or dynamic expiratory CT were performed, but the presence of air trapping in this patient would merely reinforce the already known endobronchial nature of the abnormality, whereas the lack of air trapping would not change what is already known and would probably be explained on a technical basis (suboptimal expiratory effort, inadequate timing of the expiratory effort such that the technique "missed" transient air trapping, etc.). Virtual bronchoscopy would be capable of showing the endoluminal perspective of the lesions seen at unenhanced thoracic $\mathrm{CT}$, but would not provide additional, management-altering information.

Which of the following represents the most likely diagnosis for this patient?

1. Aspirated foreign body

2. Carcinoid tumor

3. Glomus tumor of the airway

4. Mucoepidermoid carcinoma

5. Squamous cell carcinoma of the bronchus 


\section{Correct! \\ 1. Aspirated foreign body}

Given the rapid onset of symptoms and pulmonary parenchymal abnormalities in the lung parenchyma subtended by the affected airways, the complete absence of any lesion in the affected airways one year previously, and the hyperattenuating, faceted morphology of the two, distinct, endobronchial foci, aspirated foreign bodies are most likely. The involvement of the right bronchial system is also consistent with this impression. The hyperattenuating nature of the endobronchial foci raises the possibility of calcium, which can be seen with carcinoid and mucoepidermoid tumors, both of which are found in endobronchial locations, but there are causes of hyperattenuation at CT that can occur outside the context of a calcifying neoplasm. For example, nonneoplastic causes of calcification (such as infections and dystrophic calcification), metallic substances (such as iron), and plastic may all show hyperattenuation at CT imaging. The complete lack of any observable abnormality in the airways one year earlier is a strong argument against airway neoplasia as the cause of the findings in this patient.

Further clinical course: The patient underwent fiberoptic bronchoscopy which showed two endobronchial foci in the right mainstem bronchus and bronchus intermedius, consistent with foreign bodies. The lesions were extracted and found to represent two separate calcium and magnesium tablets. The airway adjacent to the foreign bodies was abraded, erythematous, and edematous, but no fixed obstruction was present).

Diagnosis: Aspirated endobronchial calcium and magnesium tablets.

\section{References}

1. Huang HJ, Fang HY, Chen HC, Wu CY, Cheng CY, Chang CL. Three-dimensional computed tomography for detection of tracheobronchial foreign body aspiration in children. Pediatr Surg Int. 2008;24(2):157-60. [CrossRef] [PubMed]

2. Medidi S, Fountain A, Radwan M, Rumbak M. "Fishing in the trachea": a unique case of foreign body aspiration. J Bronchology Interv Pulmonol. 2012;19(2):168-70. [CrossRef] [PubMed]

3. Haliloglu M, Ciftci AO, Oto A, Gumus B, Tanyel FC, Senocak ME, Buyukpamukcu N, Besim A. CT virtual bronchoscopy in the evaluation of children with suspected foreign body aspiration. Eur J Radiol. 2003;48(2):188-92. [CrossRefl [PubMed]

4. Dikensoy O, Usalan C, Filiz A. Foreign body aspiration: clinical utility of flexible bronchoscopy. Postgrad Med J. 2002;78(921):399-403. [CrossRef] [PubMed]

5. Swanson KL, Edell ES. Tracheobronchial foreign bodies. Chest Surg Clin N Am. 2001;11(4):861-872. [PubMed] 